Scientific Visualization, 2019, volume 11, number 1, pages 57 - 69, DOI: 10.26583/sv.11.1.05

\title{
Simulation and visualization of the flow around blunted body in the wake of supersonic ejecting pellet
}

\author{
Ya.V. Khankhasaeva ${ }^{1}$, A.L. Afendikov², A.E. Lutsky ${ }^{3}$ I.S. Menshov4, V.S. Nikitin5 \\ Keldysh Institute of Applied Mathematics (Russian Academy of Sciences), Moscow, Russia \\ 1 ORCID: oooo-ooo3-2748-2591, hanhyana@mail.ru \\ 2 ORCID: 0000-0002-7880-555X, andre@keldysh.ru \\ 3 ORCID: 0000-0002-4442-0571, lutsky@kiam.ru \\ 4 ORCID: 0000-0003-2300-4007, menshov@kiam.ru \\ 5 ORCID: oooo-0001-8301-3357, zogorlol@gmail.com
}

\begin{abstract}
The visual and physical features of the flow forming around spherically blunted cylinder during pellet (millimeter-sized solid body) injection towards the flow with supersonic speed are considered. The structure of this time dependent flow is very complicated and new ideas are used in the study.

Numerical simulation of moving bodies is made by the free boundary method (version of immersed boundary method) on multilevel Cartesian grids with local adaptation based on the wavelet analysis.

Dynamics of a moving body interaction with the bow shock, formation of the reverse flow region between bodies, its deformation and disappearance, and subsequent establishment of a stationary flow are studied. Reduction of main body drag to the level of $20 \%$ of the original is obtained.

In this process, several specific stages can be found. When the front part of the pellet is in the subsonic flow behind the front bow shock wave it has little effect on the outside flow. Then pellet interacts with the bow shock wave and deforms it. Recirculation zone forms between bodies. It grows to a certain size, after which, because of the pellet wake intensity lessening due to the pellet's increasing distance from the main body, it begins to decrease and is eventually blown away from the front part of the main body. Stationary flow close to the initial one (i.e., before pellet injection) is established around the main body.

Flow dynamics are illustrated by a series of images and animations which show the distribution of density and pressure, stream lines and mesh structure.
\end{abstract}

Keywords: computational fluid dynamics, free boundary method, Cartesian grids, mesh refinement, flow past moving bodies.

\section{Introduction}

Initially, pellets and systems for their injection were used in nuclear physics and energetics. Pellets are millimetersized solid bodies formed from frozen hydrogen isotopes and are used to deliver fuel to the thermonuclear reaction zone, to make hot plasma diagnostics, to control the operating conditions of the reactor, etc.
However, there exist some interesting applications of shooting small bodies (pellets) in aerodynamics. If the acceleration system (for example, a railgun) is installed in an aircraft and pellets are shot towards the flow, then it is possible to achieve a significant aircraft drag reduction $[1,2]$. This effect is associated with the formation of a narrow wake behind the pellet characterized by lower values of the total pressure, Mach number and density, which significantly affects the flow structure. 
At supersonic speeds, a complex trailing flow forms behind the body base. Close to the body base is the so-called "near wake", tapering down to its "bottleneck". At a certain distance behind it (depending on the shape of the body and the speed of flow), a distant wake is formed, characterized by lower values of Mach number, total pressure and density. The noted features of the wake flow have a significant effect on the flow structure between two consecutively placed bodies and on the flow pattern and aerodynamic characteristics.

Supersonic flow around separating bodies is being actively studied at present and a number of papers have been devoted to it, both numerical and experimental. In [3] an experimental and computational investigation of the unsteady separation behavior of two spheres is carried out. The spherical bodies, initially contiguous, are released with negligible relative velocity and thereafter fly freely according to the aerodynamic forces experienced. The qualitative separation behavior and the final lateral velocity of the smaller sphere are found to vary strongly with both the radius ratio and the initial alignment angle of the two spheres. In [4] multi-body interference during separation of a carrier model and an internal store model was investigated. The results show that the aerodynamic characteristics of the loading model change dramatically during the separation and that strong wake interference still exists even on long separation distances. In [5] an experimental, time-dependent separation of tangent bodies (plate and wedge-store) was performed to investigate the significance of transient effects and the suitability of using steady-state assumptions to predict a dynamic separation event. Dynamic motion of the wedge did not significantly affect shock-wave development between the bodies, and steadystate corrections that accounted for the motion-induced wedge angle were appropriate for predicting time-dependent surface pressures induced by the incident shock wave. However, unsteady pressures caused from the motion of the wedge were evident when separation distances were less than 20\% of the wedge width. In [2] physical experiments on pellet shooting from a blunt cylinder were conducted. It was shown that the injection of pellet leads to the significant bow shock wave transformation. After the transformation the bow shock shape is close to conical. In $[1,7,8,12]$ numerical and experimental studies of the influence of the stationary body on the one after it was conducted. Depending on the distance between bodies two distinctive flow patterns emerge. In [9-13] numerical and experimental studies of separation of the two coaxially arranged bodies were carried out. It is shown that the gas parameters in the separated flow region between the bodies depend on the rate of separation, even for a very small value of this rate in comparison with the freestream velocity. In all these works relative body movement was quite slow.

During the slow separation of the successively arranged bodies of rotation, depending on the distance between bodies two flow regimes can be observed [68]. The first regime can be observed in the initial stage of separation, when the distance between bodies is less than critical. Closed recirculation zone forms between bodies and the external flow flows around it as a solid continuation of the front body. With the distance increasing to critical closed separated flow between bodies collapses. Restructure of the flow occurs. Base flow after the front body and bow shockwave in front of the rear body form. Second flow regime forms. The analysis of various factors established [6-8] that the critical distance between the bodies, at which the restructuring of the flow occurs, is dependent on the relative size and shape of the bodies and the Mach number. In addition, the critical distance is dependent on whether it is convergence or separation process.

In applications the flows with supersonic speeds are of interest. However, in the case of fast pellet motion, the flow pat- 
tern becomes more complex and presents significant computational difficulties. In the present work, numerical simulation of the flow past a spherically blunted cylinder with a pellet emitted from it with a velocity $M=4$ in the framework of Euler equations is carried out. As [14] shows, Euler system of equations can be successfully used to model separation flows characteristic to this type of problems.

Several specific stages can be distinguished during this process. When pellet is in the subsonic flow behind the front bow shock wave and main body, it has little effect on the outside flow and main body drag. Then pellet interacts with the bow shock wave and deforms it. A recirculation region is formed between bodies. It breaks down fairly quickly into two, one behind the pellet and the other in front of the main body, due to the high pellet velocity and a large difference in the size of the bodies. Until this, main body drag increases due to the interaction of the bow shock wave from the pellet and the remnants of the bow shock wave from the main body, after which the drag begins to fall smoothly to a level of $60 \%$ of the original due to the size increase of the recirculation zone in front of the main body, which displaces the zone of increased pressure from the front of the main body. The recirculation zone grows to a certain size, after which, because of the pellet wake intensity lessening due to the pellet's increasing distance from the main body, it begins to decrease and is pushed to the main body. This is accompanied by a decrease in main body drag to a level of $20 \%$ of the original. In the end recirculation zone is blown away from the front part of the main body and main body drag increases to a level of $95 \%$ of the original. Stationary flow close to the initial one (i.e. before pellet injection) is established around the main body.

For this type of flows free boundary method in combination with local adaptation based on wavelet analysis, is quite effective $[15,16]$ and is used in the present work. A Cartesian grid is used, which covers both the region of the flow and the area occupied by the solid bodies. To fulfill the boundary condition on the body surface compensatory fluxes of mass, momentum and energy are introduced. One of the major advantages of this approach is the simplicity of grid construction which does not depend on the complexity of the body geometry.

Due to the dynamic nature of the problem it makes sense to adapt the grid to the moving features. For this purpose we use multilevel Cartesian grids with local adaptation based on wavelet analysis. For local evaluation of the smoothness of the grid function a criteria based on wavelet decomposition on local templates is applied, which allows us to clearly see the location of the discontinuities and large gradients, which in turn helps in the understanding of flow physics.

For a deeper understanding of flow physics, visualization tools such as the animation of density and pressure fields with stream traces are used to illustrate the process described above. They allow us to track the interaction of discontinuities and instabilities and demonstrate the evolution of vortices. 


\section{Problem statement}

The main body (body 2) is cylinder $70 \mathrm{~mm}$ in diameter with spherical head. Cylinder length is $100 \mathrm{~mm}$. On the axis of symmetry of the cylinder there is a channel $3 \mathrm{~mm}$ in diameter, through which the pellet (body 1) is injected into the flow. The speed of the oncoming flow is $\mathrm{M}=3$.

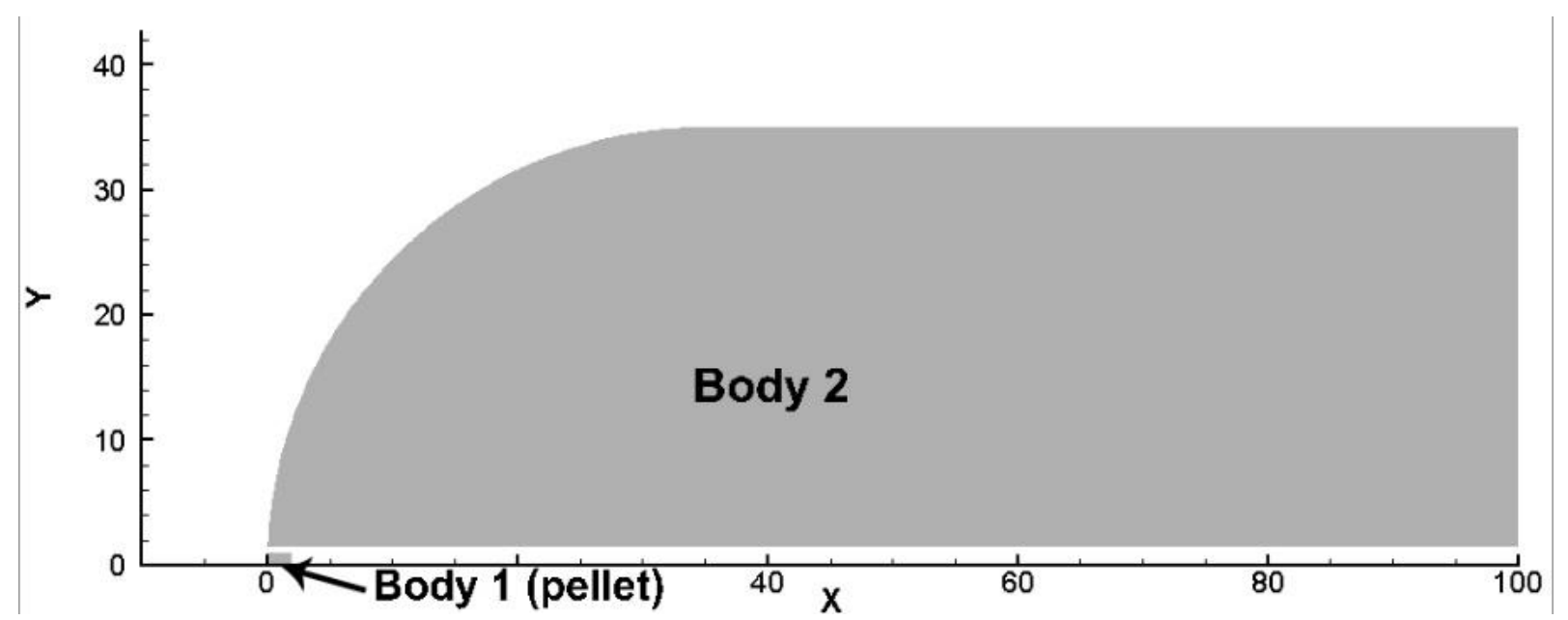

Fig. 1. Problem statement.

Pellet (body 1 ) is a cylinder $2 \mathrm{~mm}$ in diameter and $2 \mathrm{~mm}$ in length and at the start of the movement is located near the front point of body 2. It flies out of the channel of the body 2 at a speed $M=4$ (relative to the body).

All bodies are represented using the free boundary method $[15,16]$. The grid has 6 levels, i.e. dimensions of the smallest cell (6th level) are 32 times smaller than those of the largest cell (1st level). A model of the Euler equations describing the motion of an ideal compressible fluid is used which is solved by the finite volume method. The algorithm provides a second order approximation on smooth flow domains. The problem was solved in axisymmetric formulation. Numerical algorithm used is described in detail in [18] and had undergone extensive testing, see $[12,13,18]$.

\section{Graded trees and hier- archies of Cartesian grids}

In the Cartesian grid method used in the present paper, it is assumed that the problem is initially considered on a fixed zero-level grid consisting of $d$-dimen- sional cubes in which time-dependent subdivisions are constructed.

Therefore, without loss of generality, the problem of adaptation can be considered in the unit cube $\Omega \subset R^{d}$. On it a sequence of binary partitions $\left\{V_{j, k}\right\}$ is considered to be a predetermined, where index $1 \leq j \leq L$ corresponds to the level of the partition, and the multi-index $k$ corresponds to the location of the cell at this level,

$$
\left\{V_{j, k}\right\}=\left(2^{-j} k_{1}, 2^{-j}\left(k_{1}+1\right)\right) \times \ldots \times\left(2^{-j} k_{d}, 2^{-j}\left(k_{d}+1\right)\right) \text {, }
$$

where $d$ is the dimension of the space, and

$$
k_{l} \in I_{j}, \quad I_{j}=\left\{0,1, \ldots, 2^{j}-1\right\}
$$

We define the set of integer vectors $J=\{0,1\}^{d}$. Then the closure of each cell $V_{j, k}, j<L$ can be represented in the form

$$
\bar{V}_{j, k}=\bigcup_{m \in M_{j, k}} \bar{V}_{j+1, m},
$$


where $M_{j, k}$ is a set of indices defining the division of the cell $\bar{V}_{j, k}$ into cells belonging to the level $(j+1)$

$$
M_{j, k}=\{2 k+l ; l \in J\} .
$$

We call $M_{j, k}$ the refining set of indices, and the expansion (1) - a refining partition of the cell $V_{j, k}$.

Thus, each cell $V_{j+1, k}$ belonging to the level ${ }^{j+1}$ is included in the refining partition of some cell $V_{j, l}$ of level $j$. Cell $V_{j+1, k}$ is called the descendant of the cell $V_{j, l}, V_{j, l}$ is in turn is called the ancestor of $V_{j+1, k}$.

Definition: A graded Cartesian tree is a partition

$\bar{\Omega}=\bigcup_{(j, k) \in \mathfrak{I}^{n}} \bar{V}_{j, k}, \quad \mathfrak{L}^{n} \in\left\{(j, k), \quad j=0, \ldots, L ; k \in I_{j}^{d}\right\}$,

that

1. $V_{j, k} \cap V_{i, l} \neq \varnothing \Leftrightarrow(j, k)=(i, l)$

2. If $(j, k) \neq(i, l) \quad$ and

$\operatorname{dim}\left(\bar{V}_{j, k} \cap \bar{V}_{i, l}\right)=d-1$, then $|i-j| \leq 1$

3. If $(j, k) \in \mathfrak{L}^{n}$ and $(j, k) \in M_{j-1, k}$, then $M_{j-1, k} \subset \mathfrak{L}^{n}$

In other words, each cell from the hierarchical structure enters the tree only together with all the elements of the refining partition of the corresponding cell of the previous level.

Such objects appeared in works on harmonic analysis, beginning with the classical works of Hardy-Littlewood [17].

In calculations, each cell of the index $(j, k)$ has a flag indicating whether the given cell is the calculated and final (i.e., one that participates in the work of the solver) or subdivided into cells of the next level. For a subdivided cell, there are pointers to the descendants of this cell. For a final cell, these pointers are empty, but there is a pointer to an array that describes the real physical cell. It contains the coordinates of the cell and the vector of gas-dynamic parameters inside it. In case the cell is subdivided this pointer is zero.

For automatic local refinement and coarsening of the grid in accordance with the local flow properties, a grid analyzer is used based on the calculation of the coefficients of expansion on a wavelet basis constructed from Bsplines.

In the computational algorithm traversal of the $d$-dimensional array of trees described above, is carried out in cycles along all elements of the basic grid. In the case where the current cell is final, the target function is applied to it, otherwise the procedure is repeated recursively for all the descendants of this cell. To search for neighbors of the given cell, property 3 of the graded tree is used; a cell of the $j$ level with an index $\left(k_{1}, k_{2}\right)$ has as its parent a cell ${ }^{j-1}$ of a level with indices $\left(\left[k_{1} / 2\right],\left[k_{2} / 2\right]\right)$, where brackets denote the taking of the whole part. So, for example, if there are only two levels, the neighbors of the current cell for each of the axes can be obtained by a simple search based on half division and multiplication of the indices.

When working with multilevel computational grids, there is a need for indicators that signal the need to rebuild the grid. One of the possible options for constructing such indicators is given in [12-14]. In this paper we use a more efficient analyzer on an irregular grid, the principles of construction and properties of which will be given in a separate publication. 


\section{Computation results on Cartesian grids with local adaptation}
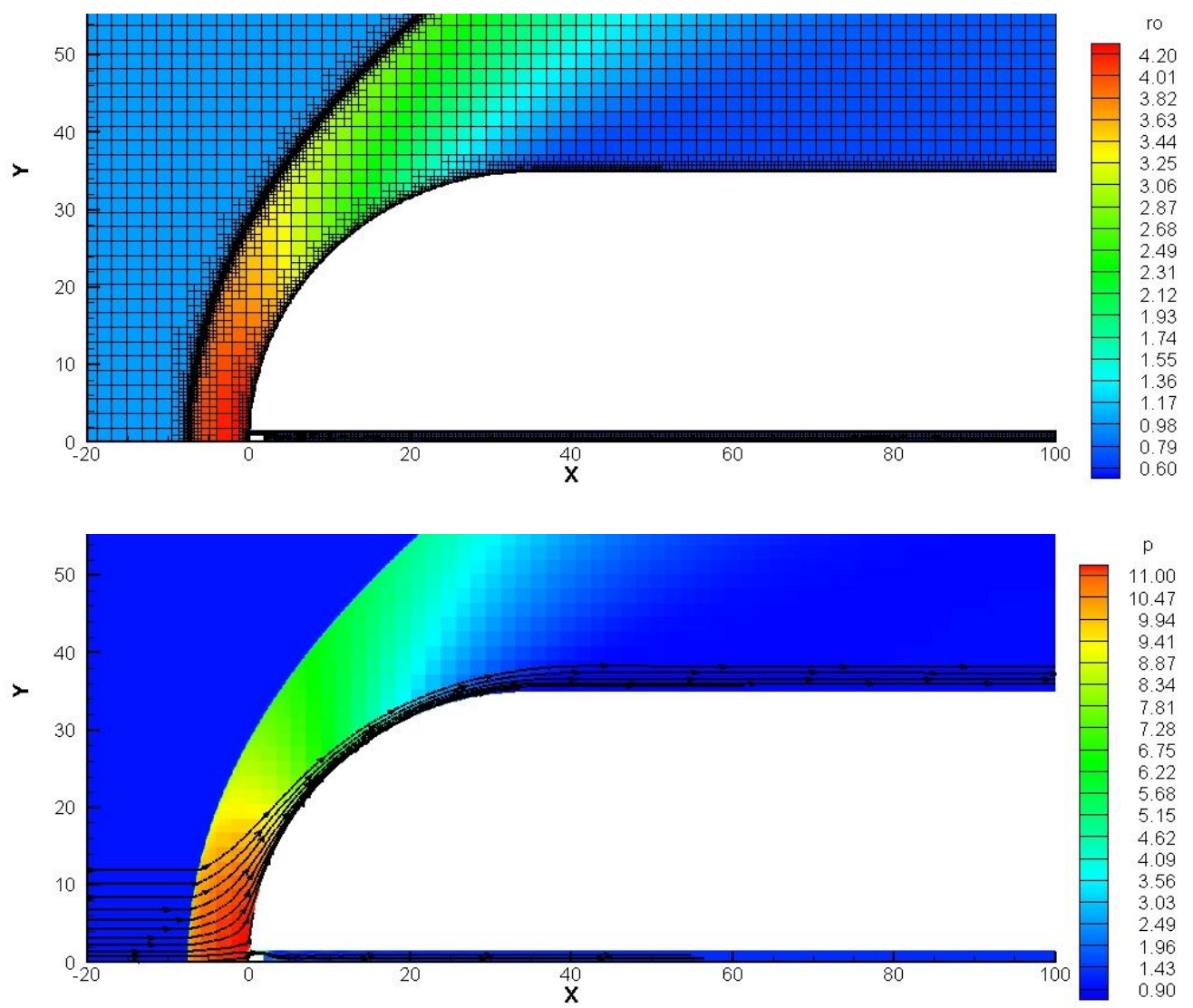

Fig. 2. Density distribution and mesh structure (top) and pressure distribution with stream traces (bottom) at the start of pellet motion.

Mesh adaptation to the solution is conducted based on density field analysis. As Fig. 2 shows, wavelet-analysis captures discontinuities (a strip of small cells at the shock front). The unperturbed flow does not require adaptation, therefore, outside the bow shock the cells are of the base level (the biggest size). For the best approximation of the geometry on the boundary of the body cells have the maximum level (the smallest size). The external flow forms a system of reflected shocks in the channel (Fig. 3).

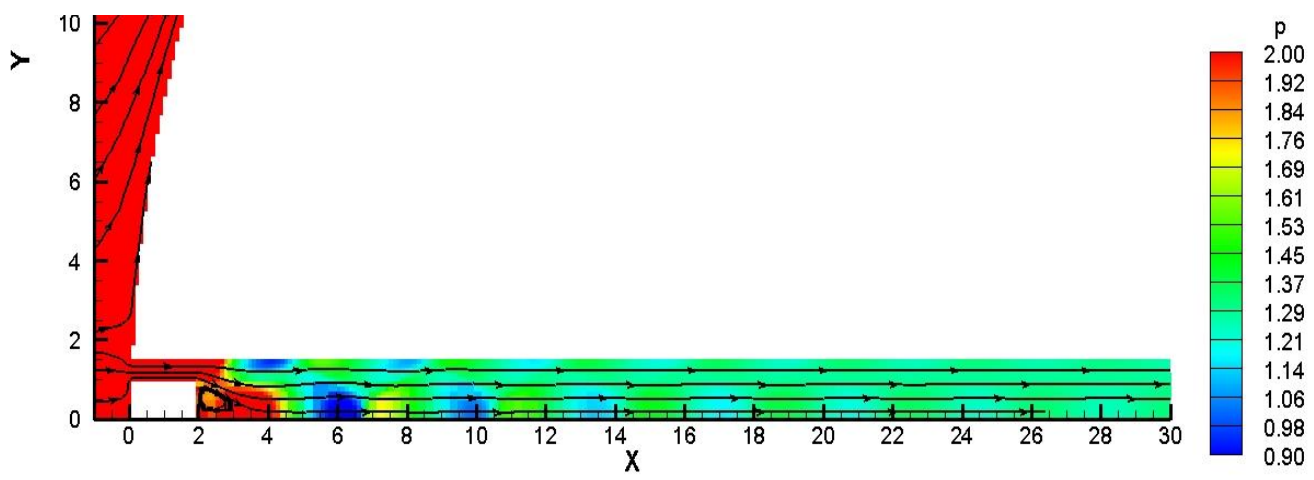

Fig. 3. Pressure distribution with stream traces in channel.

The body 1 is accelerated in such a way that when it crosses the front of the bow shock wave it has a velocity $\mathrm{M}=4$. In Fig. 4 instantaneous flow patterns at various stages of pellet injection are presented. 

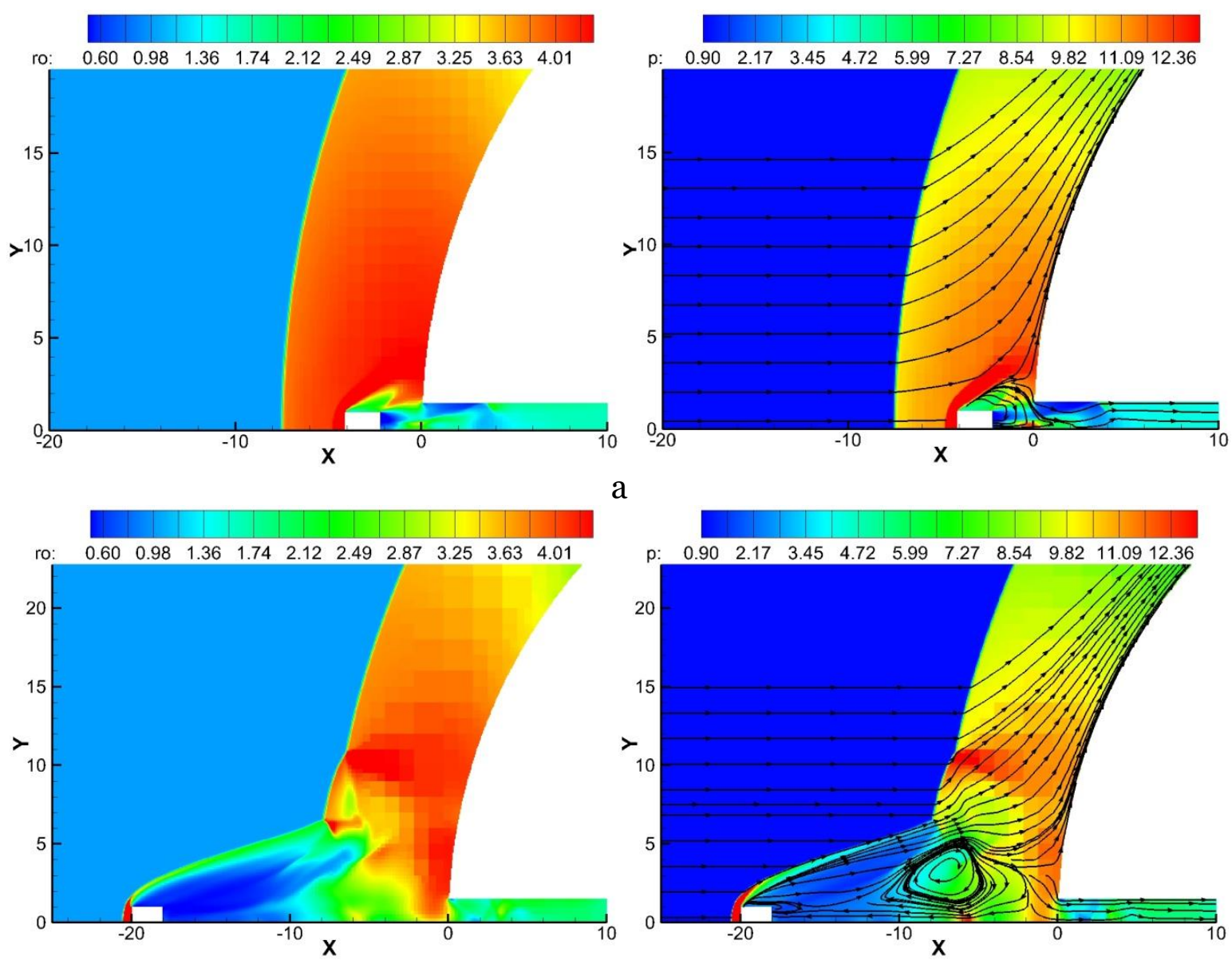

Fig. 4. Density (left) and pressure (right) distributions with stream traces at different times of pellet motion, $\mathrm{a}-\mathrm{t}=2, \mathrm{~b}-\mathrm{t}=5(\mathrm{t}=\mathrm{O}-$ start of pellet motion).

The general flow pattern is similar to that described in [13], where front body was moving with much lower speed but there are also significant differences.

On Fig. 4a pellet (body 1) is in the subsonic flow behind the front bow shock wave and main body. A recirculation zone starts to form between bodies 1 and 2 and external flow flows around the body 1 and 2 as a whole. Since the body 1 moves with supersonic speed, typical supersonic flow configuration begins to establish around it, i.e., a bow shock wave, a rarefaction fan, base flow. At this moment main body (body 2) drag changes little (Fig. 5).

Then pellet crosses the bow shock wave and deforms it (Fig. 4b). Bow shock wave from pellet and bow shock wave from main body interact with each other, forming a Mach configuration. Between time moments presented on Fig. 4a and b recirculation zone grows in size until it fills the whole space between the body 1 and 2 . At $t=5$ on Fig. $4 \mathrm{~b}$ recirculation zone breaks down into two, one behind the pellet and the other in front of the main body, due to the high pellet velocity, distance and a large difference in the size of the bodies. Behind body 1 its own base flow is established. As the pellet moves with supersonic speed, the flow inside the recirculation zone in front of the main body also has supersonic speed. The flow at the upper point of the recirculation zone is braked by the main body, increasing the pressure on its surface and its drag (Fig. 5).

Because recirculation zone at this stage still continues to grow in size filling an increasing part of the front surface of the main body, its drag will decrease and reach the level of $60 \%$ of the original. As the recirculation zone grows slower than the pellet moves, then because of pellet's increasing distance from the main body and its wake intensity lessening, the growth of the recirculation region will gradually slow down, which explains the drag coefficient curve in Fig. 5 at t from 20 to 90. 


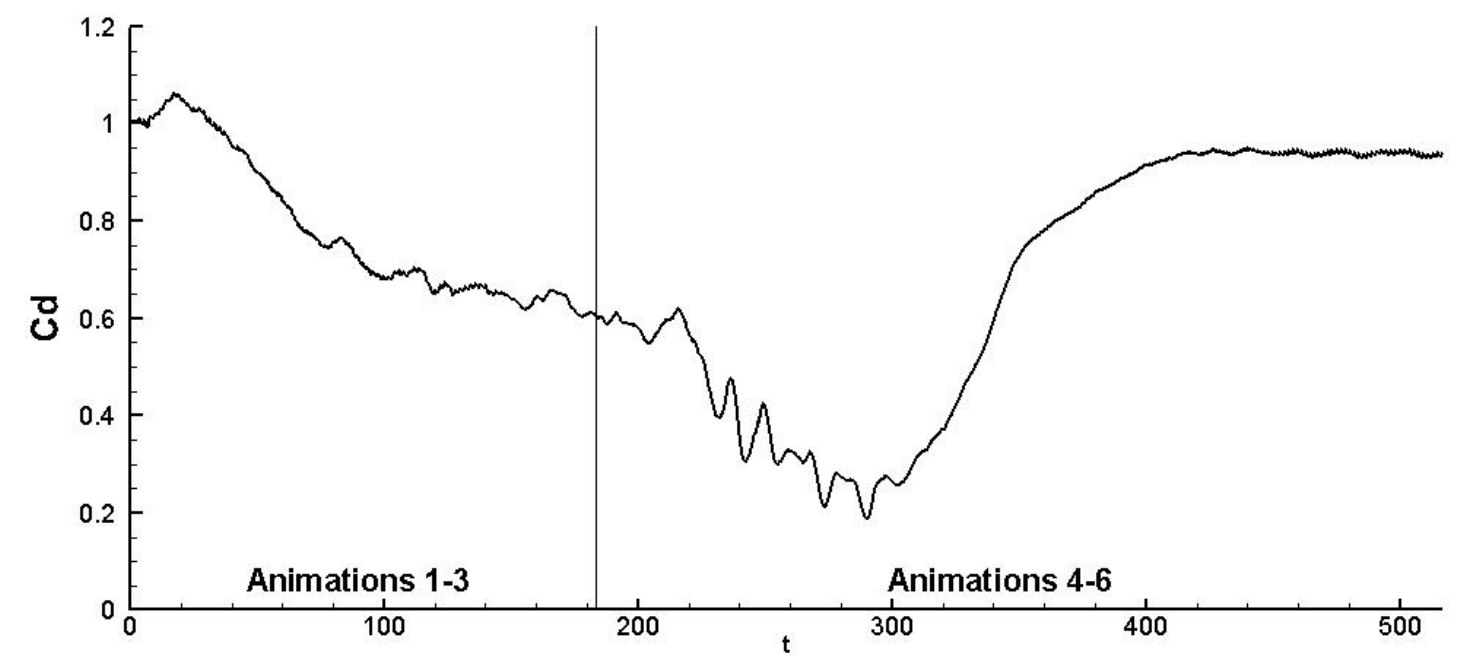

Fig. 5. Drag coefficient of main body (body 2) during the pellet movement.
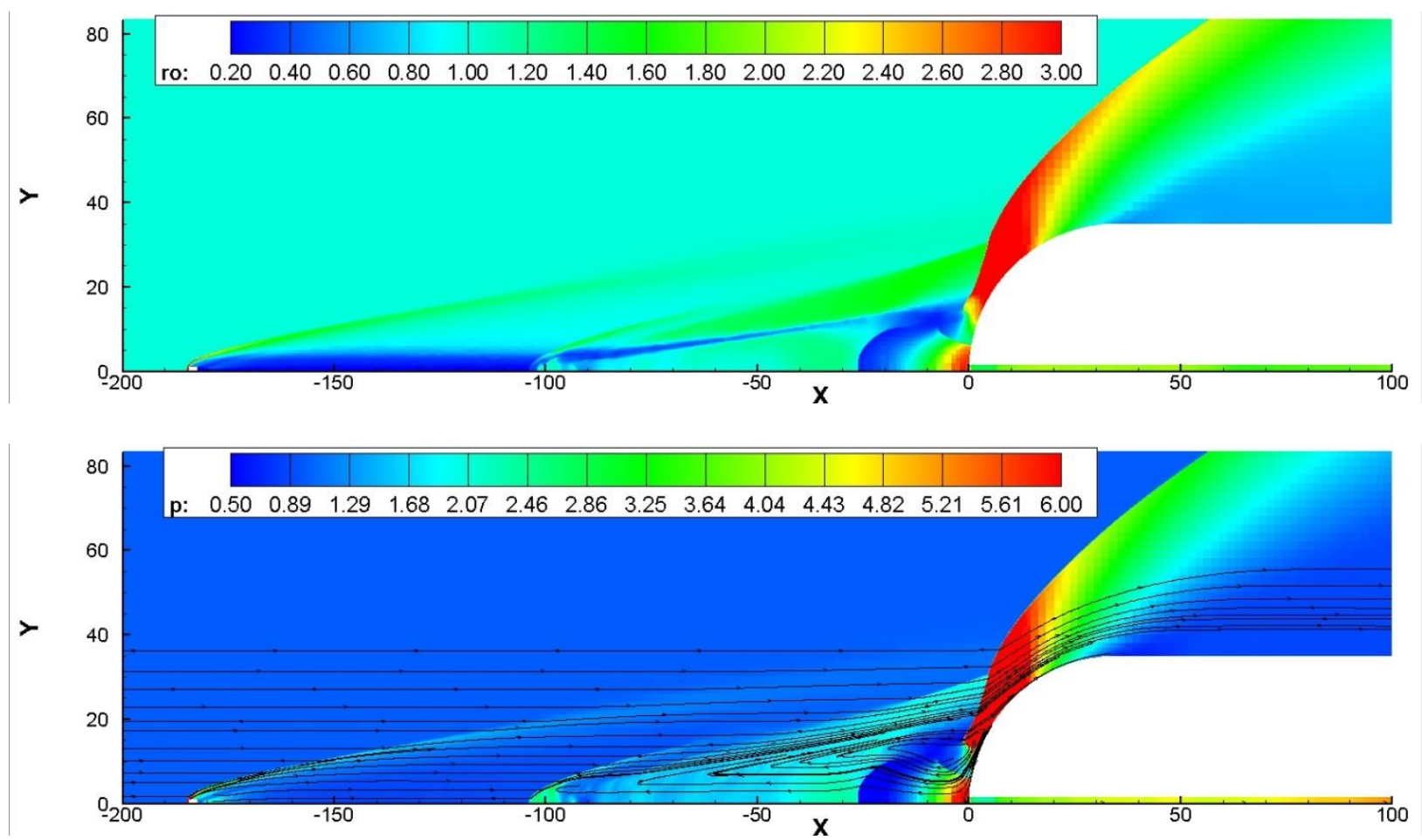

Fig. 6. Density (top) and pressure (bottom) distributions with stream traces at $t=40.5$.

On Fig. 6 recirculation zone continues to grow in size. Pellet has moved far enough away that a shock wave has formed from the recirculation region, which also forms a Mach configuration with a shock wave from the main body. Since flow inside the recirculation zone is supersonic, shock waves are formed inside it, when the flow is decelerated. 

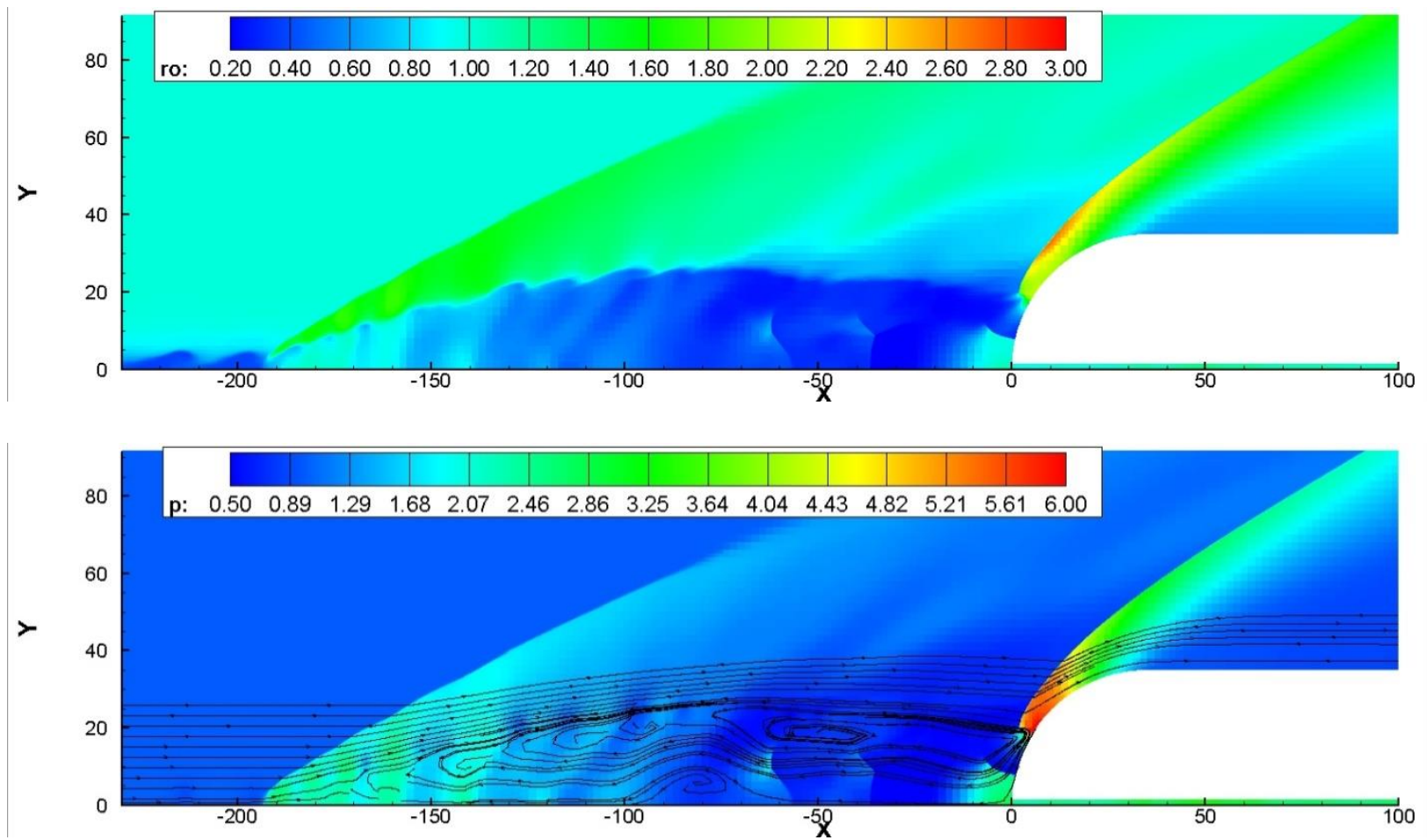

Fig. 7. Density (top) and pressure (bottom) distributions with stream traces at $\mathrm{t}=184.5$.

On Fig. 7 pellet has moved so far that, firstly, the Kelvin-Helmholtz instability begins to appear, both in the wake after the pellet and at the recirculation zone's upper boundary, and secondly, the recirculation zone ceases to grow, and its leading edge stops at $\mathrm{x}=\mathrm{-}$ 200, after which it will move back to the main body. Pellet wake instability affects the recirculation zone's flow structure, generating oscillations propagating in the direction of the main body. They are responsible for the oscillations in the drag on Fig. 5.
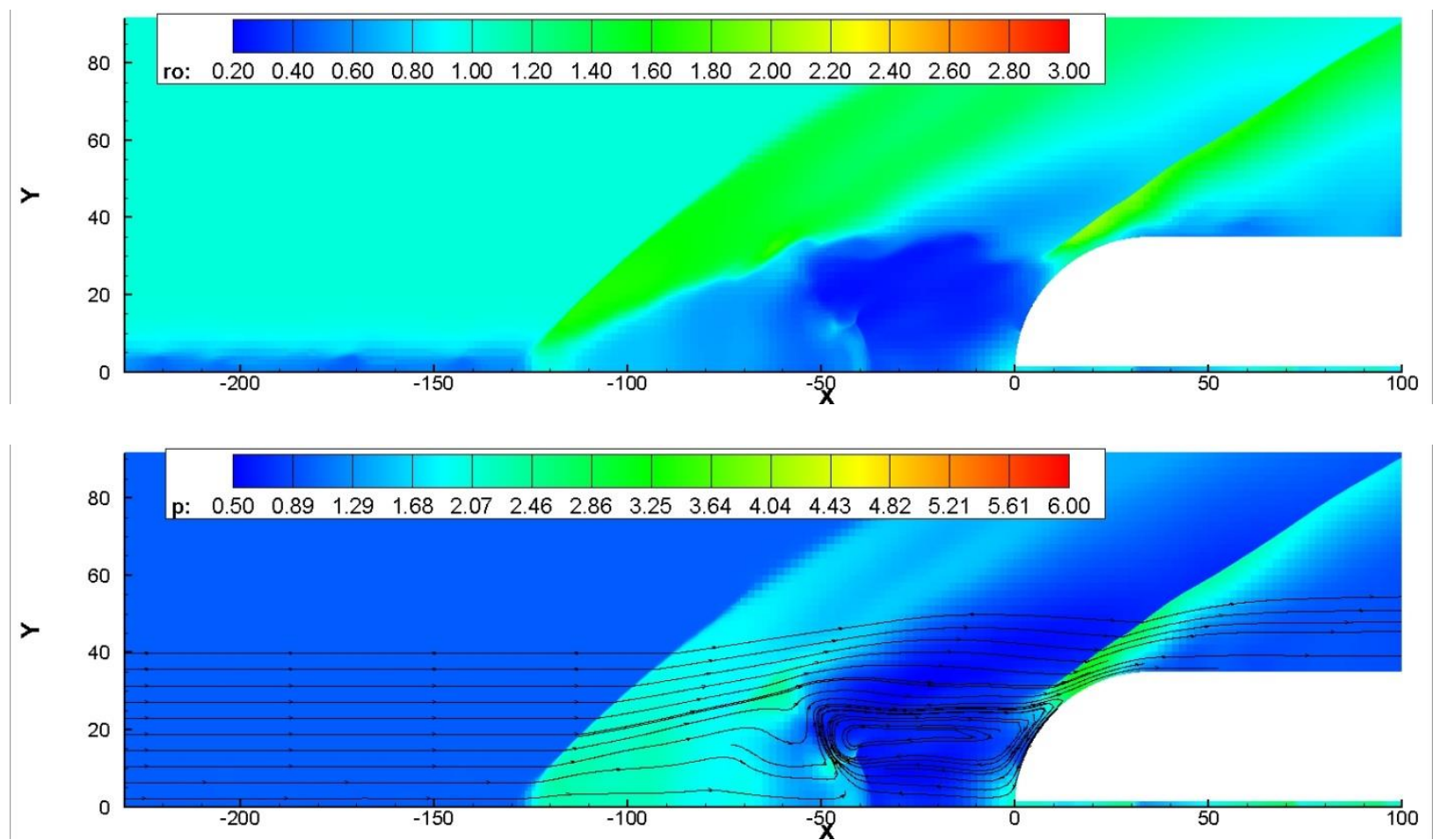

Fig. 8. Density (top) and pressure (bottom) distributions with stream traces at $\mathrm{t}=264.5$. 
Due to pellet's increasing distance from the main body and resulting lessening of its wake intensity, the recirculation zone loses its stability and starts to decrease in size (Fig. 8). Under the action of a shock wave, it shifts to the main body, while covering an even larger portion of its surface and shifting the zone of increased pressure on it, thereby reducing main body drag to a level of $20 \%$ of the original (Fig. 5 ).
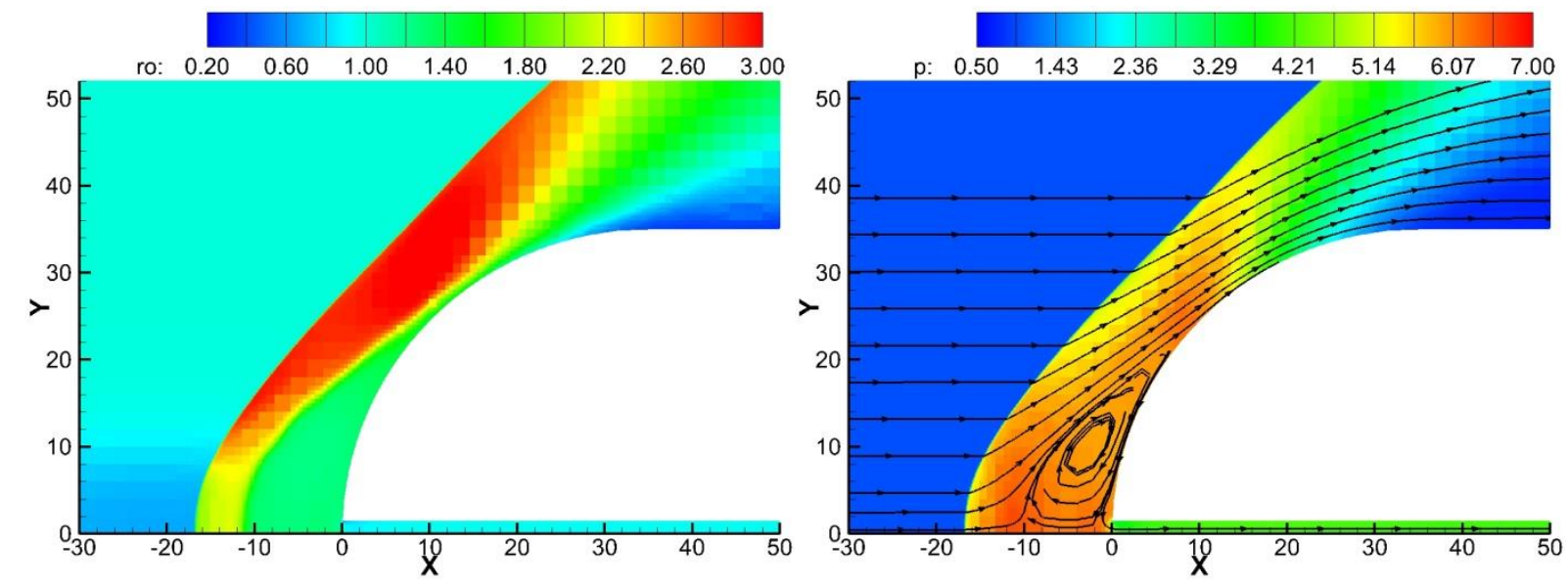

a
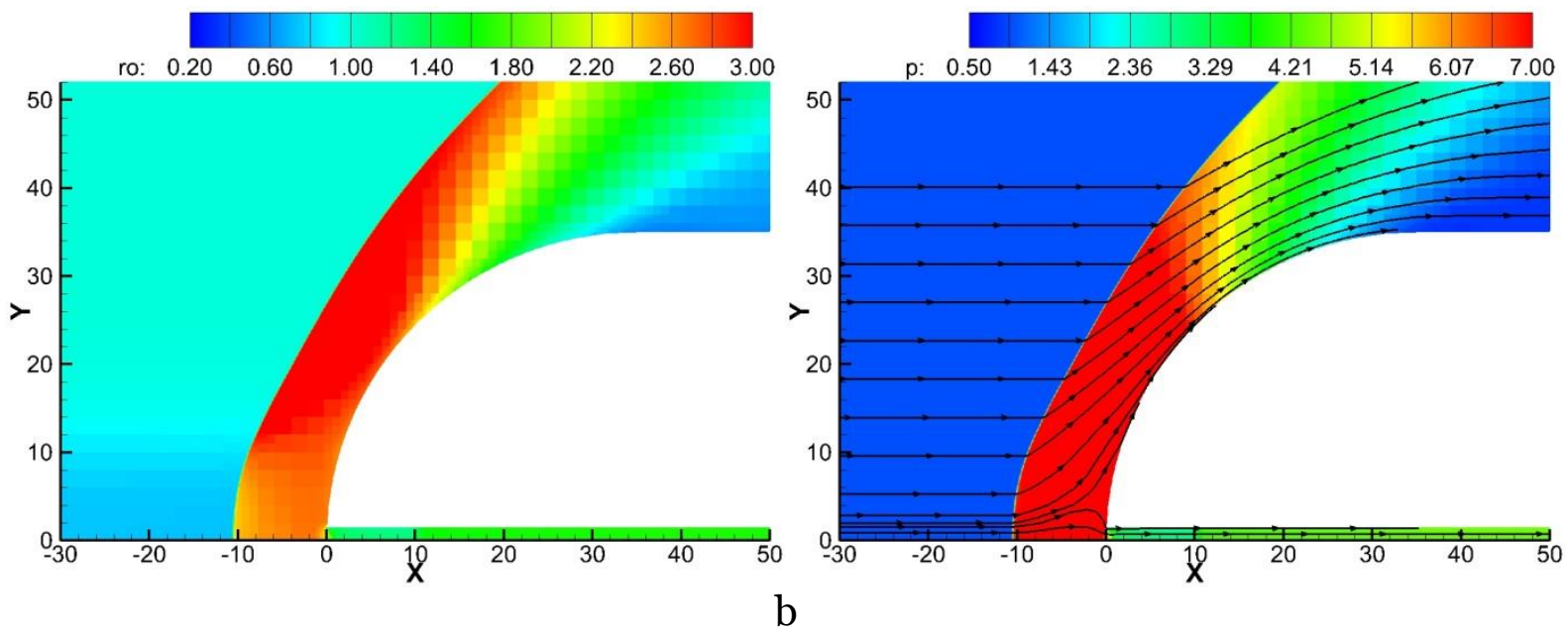

Fig. 9. Density (left) and pressure (right) distributions with stream traces at different times, $\mathrm{a}-\mathrm{t}=376, \mathrm{~b}-\mathrm{t}=516$.

In the end of the process the recirculation zone is blown away from the head of the main body, part of it goes through the channel, another along the lateral surface of the main body. Flow close to a supersonic $(\mathrm{M}=3)$ freestream flow around only main body (a spherically blunt cylinder) is established. Main body drag increases and reaches a level 95\% of the original.

Resulting pellet shooting process coincides with the experiment described in [2], from which part of the problem formulation (speed and dimensions of the pellet, dimensions of the channel and the main body) is taken. The same flow characteristics as the interaction of the pellet with the bow shock wave and its subsequent deformation, as well as its return to the initial form, are noted. Just as in [1], where the flow past a stationary pellet and a spherically blunted cylinder with $\mathrm{M}=5$ located behind it at a certain distance was studied, a substantial decrease in the main body drag was obtained.

For a deeper understanding of the physics of the flow, animations of density and pressure fields with current lines and grid restructuring throughout the entire process described above are presented. 
Animations of gas-dynamic fields make it possible to track the interaction of discontinuities and instabilities. Animations with stream traces demonstrate the evolution of vortices. As can be seen from the mesh restructure animations, the wavelet analysis captures discontinuities well. The grid has a maximum (6th) level on all shocks and contact discontinuities, at the border of the wake and the recirculation zone.

\section{Conclusion}

Supersonic pellet ejection from the channel of a spherically blunted cylinder towards supersonic inflow has been numerically investigated with the Cartesian grid method implemented with the free boundary method for treating boundary conditions on moving solid surfaces and the local wavelet grid adaptation for enhancing grid resolution.

In the process of calculating the nonstationary flow around the blunt body and the pellet, the grid adaptation has been performed to the moving body (pellet) and to regions of steep gradients recognized by the wavelet analysis. The grid adaptation has been shown to increase the solution accuracy with significant savings in computational time; similar accuracy on a non-adaptive uniform fine grid would take an order of magnitude more time.

Visualization tools (instantaneous images and animations of gas-dynamic fields and stream traces) have been employed which provide deep insight into the nature and dynamics of non-stationary wake flow and complex shock interaction processes.

Due to complexity of the problem and its great practical interest, further more detailed calculations are required. First of all, it is necessary to investigate dynamic effects and, in particular, pressure pulsations.

\section{Acknowledgements}

This work was supported by Russian Science Foundation, project 17-7130014 .

\section{References}

1. S. Leonov, V. Bityurin, A. Yuriev, S. Pirogov, B. Zhukov. "Problems in Electric Method of Drag Reduction and Flow/Flight Control", AIAA2003-0035, 41th AIAA Aerospace Sciences Meeting \& Exhibit, Reno, NV, USA, 2003.

2. Erofeev, Tatiana Lapushkina, Serguei Poniaev, Roman Kurakin, and Boris Zhukov. "Flow Around Different Bodies at the Pellet or Plasma Jet Injection", 50th AIAA Aerospace Sciences Meeting including the New Horizons Forum and Aerospace Exposition, 2012

3. S. J. Laurence, N. J. Parziale and R. Deiterding. Dynamical separation of spherical bodies in supersonic flow. J. Fluid Mech. (2012), vol. 713, pp. 159-182. doi:10.1017/jfm.2012.453

4. Wang YuanJing. Wang Hongbia, Qian Fengxue, Chang Lixia. Investigation on Separation Interference of Typical Multi-Body Vehicle in Supersonic Conditions. 2013 Fourth International Conference on Digital Manufacturing \& Automation.

5. Neal A. Mosbarger, Paul I. King. Time-Dependent Supersonic Separation of Tangent Bodies. Journal of Aircraft. Vol. 33, No. 5, 1996.

6. Petrov K.P. Aerodinamika tel prosteishikh form [Aerodynamics of simplest forms bodies]. Izdatelstvo «Faktorial», 1998, 432 s., ISBN 588688-014-3. [In Russian]

7. Khlebnikov V.S. The pattern and restructuring of supersonic flow past a pair of bodies. Fluid Dynamics. January 1994. vol. 29, no. 1, pp.123128.

8. Khlebnikov V.S. Perestroika techeniya mezhdu paroi tel, odno iz kotorykh raspolozheno $\mathrm{v}$ slede drugogo, pri sverkhzvukovom obtekanii [Restructuring of the flow between the pair of bodies, one of which is situated in the wake of another at supersonic flow]. Uchenye zapiski TsAGI. 1976. Vol. 7. No. 3. pp. 133-136. [In Russian] 
9. V. N. Kudryavtsev, A. Ya. Cherkez, and V. A. Shilov. Study of supersonic flow about two separating bodies. Izv. AN SSSR. Mekhanika Zhidkosti i Gaza, Vol. 4, No. 2, pp. 91-99, 1969

10. R. I. Vinogradov, V. V. Trofimov, I. R. Yakubov. Osobennosti sverkhzvukovogo obtekaniya dvukh tel i ikh aerodinamicheskie kharakteristiki $\mathrm{v}$ rezhime razdeleniya [Features of supersonic flow past a pair of bodies and their aerodynamic characteristics during separation]. Uchenye zapiski TsAGI, T. 20, № 4, 102-7.[In Russian]

11. Vasenev L.G., Vnuchkov D.A., Zvegintsev V.I., Lukashevich S.V., Shiplyuk A.N. Aerodynamic drag measuring for two consistently located axisymmetrical models during their separation. $16^{\text {th }}$ International conference on the methods of aerophysical research, August 19-25, 2012, Kazan, Russia, Proceedings.

12. Lutsky, A.E., Menshov, I.S. Khankhasaeva, Y.V. The effect of incident flow on a supersonic circumfluence of a blunt object. Math Models Comput Simul (2017) 9: 92.

13. L. Afendikov, Ya. V. Khankhasaeva, A. E. Lusky, I. S. Menshov, K. D. Merkulov. "Computation and visualization of flows past bodies in mutual motion" // Scientific Visualization, 2016, T.8. № 4. C. 128-138. (URL: http://sv-journal.org/20164/10.php?lang=ru)

14. Babarykin K.V., Kuz'mina V.E., Tsvetkov A.I. Avtokolebaniya pri natekanii ravnomernogo sverkhzvukovogo potoka na telo s vystupayushei ostroi igloi [Self-oscillations during uniform supersonic flow past a body with a protruding sharp needle]. Aerodinamika. SPb.: Izdatel'stvo SPbGU. 2001. pp. 128-149. [In Russian]

15. Menshov I.S., Kornev M.A. Freeboundary method for the numerical solution of gas-dynamic equations in domains with varying geometry. Mathematical Models and Computer
Simulations, 2014, vol. 6, No. 6, pp.612-621.

16. S. Menshov and P. V. Pavlukhin, Efficient Parallel Shock-Capturing Method for Aerodynamics Simulations on Body-Unfitted Cartesian Grids. Computational Mathematics and Mathematical Physics, 2016, Vol. 56, No. 9, pp. 1651-1664.

17. E. Stein. Harmonic analysis realvariable methods, orthogonality, and oscillatory integrals // Princeton Univ. Press, Princeton, NJ, 1993.

18. Afendikov A.L., Davydov A.A., Menshov I.S., Merkulov K.D., Plenkin A.V, Khankhasaeva Ya.V. Adaptivnye veivletnye algoritmy dlya resheniya zadach gidro- i gazovoi dinamiki na dekartovykh setkakh [Adaptive wavelet algorithms for solving problems of hydro- and gas dynamics on Cartesian grids]. M.: IPM im. M.V. Keldysha, 2016. - 232 s. ISBN 9785-98354-030-9. [In Russian] 